> L'un des défis majeurs des neurosciences contemporaines est d'établir des relations entre le fonctionnement du cerveau et l'éducation. En apportant des indications sur les capacités et les contraintes du «cerveau qui apprend », les neurosciences peuvent aider à expliquer pourquoi certaines situations d'apprentissage sont efficaces, alors que d'autres ne le sont pas. Les résultats présentés ici apportent les premiers éléments d'une neuropédagogie du raisonnement. <

\section{Imagerie et cognition (6) Imagerie cérébrale, cognition et pédagogie}

Olivier Houdé

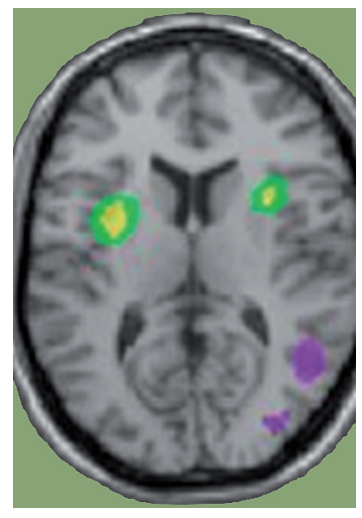

Université Paris Descartes :

Institut universitaire de France, chaire de sciences des apprentissages, UMR CNRS 6232 (CI-NAPS) : Sorbonne, laboratoire Alfred Binet, 46, rue Saint-Jacques, 75005 Paris. olivier.houde@parisdescartes.fr
Les neurosciences et l'imagerie cérébrale en particulier apportent indiscutablement des clés pour le progrès de l'Homme dans son ensemble [1]. C'est déjà le cas dans le domaine médical pour beaucoup d'exemples, un peu dans le domaine de l'économie, et ce sera le cas, peut-être plus encore, dans le domaine de l'éducation [2-6]. Bien audelà de la santé, aucun secteur de la société et de la vie psychologique des individus n'échappe au label «neuro » qu'on s'en réjouisse ou qu'on le déplore: neuroéconomie, neuropédagogie, etc., sont des nouveaux champs d'exploration de la psychologie expérimentale contemporaine [7]. Tout cela soulève bien entendu des problèmes éthiques, déjà minutieusement analysés dans le domaine biomédical [8], mais qui peuvent se poser aussi, avec force, dans le domaine de l'éducation où la réflexion (bioéducative) reste à mener. Quoi qu'il en soit et sans céder à une vision trop scientiste et naïve, voire idéologiquement dangereuse, d'une technoscience de l'éducation parfaitement contrôlée et contrôlable, on ne peut refuser l'idée qu'une recherche pédagogique nouvelle, exploitant les ressources actuelles de l'imagerie cérébrale, puisse éclairer certains mécanismes neurocognitifs élémentaires d'apprentissage dont dépendent des phénomènes éducatifs, sociaux et culturels plus complexes.

\section{Apprentissages cognitifs et cerveau}

L'apprentissage est une modification de la capacité à réaliser une tâche sous l'effet d'une interaction avec l'environnement. Dès la naissance, le bébé est géné- tiquement programmé pour apprendre. II est par exemple capable de reproduire par imitation des modèles (mouvements du visage ou des mains) que lui présente l'adulte, ce qui montre que son cerveau est d'emblée construit pour être réceptif à l'apprentissage culturel humain. Pour l'essentiel, on peut considérer qu'il existe, chez l'adulte comme chez l'enfant, deux formes complémentaires d'apprentissages cognitifs : I'automatisation par la pratique et le contrôle par l'inhibition [7].

L'imagerie cérébrale fonctionnelle a permis de montrer que dans le premier cas de figure, c'est initialement la partie préfrontale (avant) du cerveau qui est activée car la mise en place des habiletés nécessite un contrôle exécutif et un effort cognitif (apprendre par cœur une liste de mots présentée visuellement par exemple), puis ces habiletés s'automatisent avec la pratique et c'est la partie postérieure du cerveau, ainsi que les régions sous-corticales, qui prennent le relais [9-10]. Dans le cas inverse (désautomatisation), il s'agit d'apprendre à inhiber les automatismes acquis pour changer de stratégie cognitive. L'imagerie cérébrale a permis de montrer le changement qui s'opère dans notre cerveau lorsque, sous l'effet d'un apprentissage, on passe, au cours d'une même tâche de raisonnement, d'un mode perceptif facile, automatisé mais erroné, à un mode logique difficile et exact. Les résultats indiquent dans ce cas un basculement très net des activations cérébrales, de la partie postérieure du cerveau au cortex préfrontal [4, 11, 12] - dynamique cérébrale inverse de l'automatisation.

Le premier type d'apprentissage, l'automatisation par la pratique, correspond aux connaissances générales, bien établies, apprises par la répétition, la mémorisation, et qui doivent être connues de tous (les programmes à l'école par exemple). C'est ce que l'on appelle l'intelligence cristallisée par la culture. À l'inverse, et de manière complémentaire, le second type d'apprentissage, le contrôle par l'inhibition, 
fait appel à l'imagination, à la capacité à changer de stratégie de raisonnement en inhibant les automatismes habituels (eux-mêmes cristallisés). C'est l'intelligence fluide de notre cerveau [13]. Apprentissage et intelligence sont donc étroitement liés via la culture et le raisonnement.

\section{L'apprentissage par l'inhibition}

Voici une description, un peu plus détaillée, de l'apprentissage par l'inhibition, forme d'apprentissage cognitif (ou plus exactement «métacognitif ») explorée ces dernières années par la neuropédagogie expérimentale et absente des programmes scolaires. II s'agit dans ce cas d'étudier un mécanisme particulier de correction d'erreurs dans le cerveau humain. La méthode scientifique idéale est ici l'apprentissage expérimental, c'est-à-dire une sorte de «pédagogie de laboratoire » : par exemple, apprendre à l'individu (et à travers lui à son cerveau) à inhiber ses erreurs systématiques (ou biais) de raisonnement. Les questions d'apprentissage et de plasticité neurocognitive, essentielles en psychologie de l'enfant et de l'adolescent [14], le sont aussi en neuroscience cognitive. Comme l'a écrit Michael Posner au début des années 2000, «nous serons proches de l'intégration de l'esprit et du cerveau quand les questions de la circuiterie et de la plasticité des réseaux des aires anatomiques qui sous-tendent la cognition deviendront des caractéristiques toujours plus fortes des études de neuro-imagerie » [15] (voir aussi [16]). Pour l'exemple des erreurs de raisonnement logique, notre hypothèse de travail a été que la difficulté que rencontrent les individus peut tenir, dans certaines situations pièges, au fait que deux stratégies de raisonnement entrent en compétition et se télescopent dans leur cerveau, l'une perceptive (ou sémantique selon les cas : une croyance sur le monde), l'autre logique. Face à cette compétition neuronale, les élèves échoueraient à inhiber la stratégie perceptive (ou sémantique), sans qu'il s'agisse pour autant d'un problème d'absence de logique mentale (I'une des deux stratégies) en tant que telle. Afin de le démontrer, nous avons tout d'abord testé, par des études de psychologie expérimentale, l'efficacité de différentes conditions d'apprentissage sur des groupes indépendants: (1) l'inhibition d'une stratégie perceptive par des instructions exécutives venant d'autrui (un professeur qui faisait

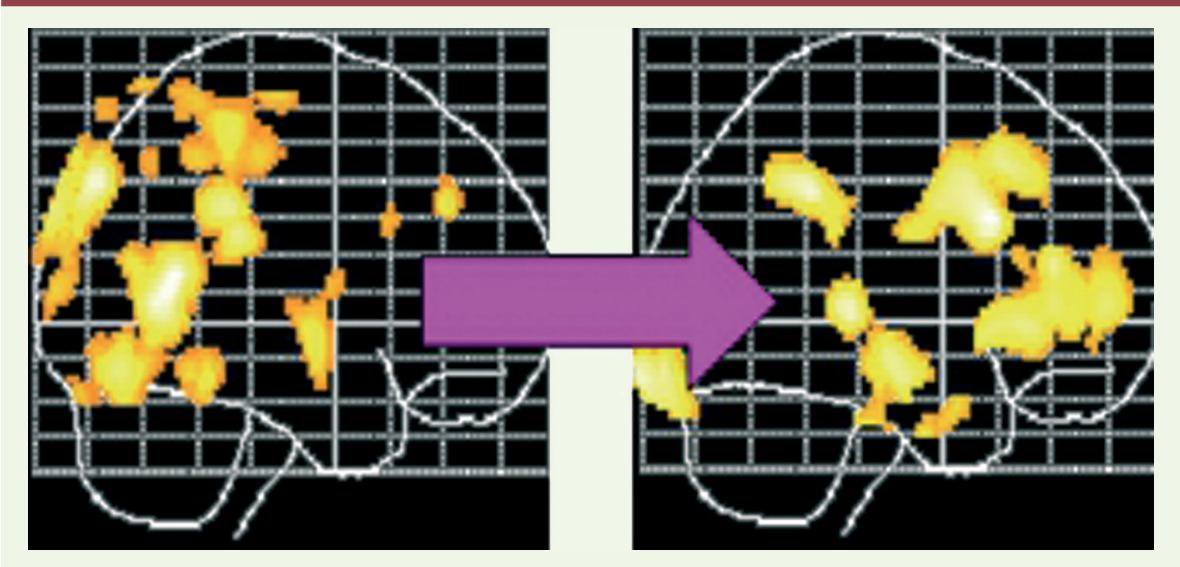

des mises en garde très ciblées sur le danger du piège perceptif à éviter, sur l'erreur possible; apprentissage dit «métacognitif » car portant sur les stratégies cognitives à sélectionner); (2) l'explication cognitive strictement logique et rationnelle du problème (venant du même professeur); et (3) la simple répétition de la tâche (ce dernier type d'apprentissage correspondant aux effets de la pratique). Seul l'apprentissage de l'inhibition s'est révélé efficace, c'est-à-dire source de progrès cognitifs transférables dans des problèmes logiques du même type : le taux de réussite, initialement inférieur à $10 \%$, est devenu supérieur à $90 \%$. Ceci indique que c'est bien ce mécanisme exécutif de blocage (inhibition) qui faisait psychologiquement défaut aux individus interrogés et non pas la logique formelle en tant que telle ou la pratique (comme c'est le cas pour d'autres situations d'apprentissage). Dans ces deux dernières conditions, le taux d'erreur est resté comparable au niveau initial face à des problèmes du même type.

Constatant ces effets psychopédagogiques différenciés en faveur du processus exécutif d'inhibition, nous avons alors repris la même expérience en imagerie cérébrale fonctionnelle afin d'observer ce qui se passe dans le cerveau des élèves avant et après l'apprentissage de l'inhibition de la stratégie perceptive, c'est-à-dire avant et après la correction de l'erreur de raisonnement. La question était de savoir s'il existe une réalité biologique, neuronale (et laquelle) à cet impact pédagogique provoqué et observé de manière comportementale. Les participants étaient donc introduits deux fois dans la caméra d'imagerie cérébrale, l'apprentissage (ou séquence pédagogique expérimentale) avec le professeur étant réalisé hors caméra. Les résultats ont montré une spectaculaire reconfiguration - ou plasticité - des réseaux cérébraux, de la partie postérieure du cerveau à sa partie antérieure, dite «préfrontale» $[4,11,12]$ (Figure 1). Cette expérience illustre ainsi de façon dynamique comment peut se mettre en place, sous l'effet d'une forme particulière de pédagogie expérimentale, un processus

Figure 1. Exemple de neuropédagogie expérimentale chez des sujets sains. Reconfiguration des réseaux cérébraux, de la partie postérieure du cerveau à sa partie préfrontale, avant (à gauche) et après (à droite) un apprentissage de l'inhibition d'un mode perceptif facile, automatisé mais erroné pour acquérir un mode logique difficile et exact lors d'une tâche de raisonnement (d'après [11]). 


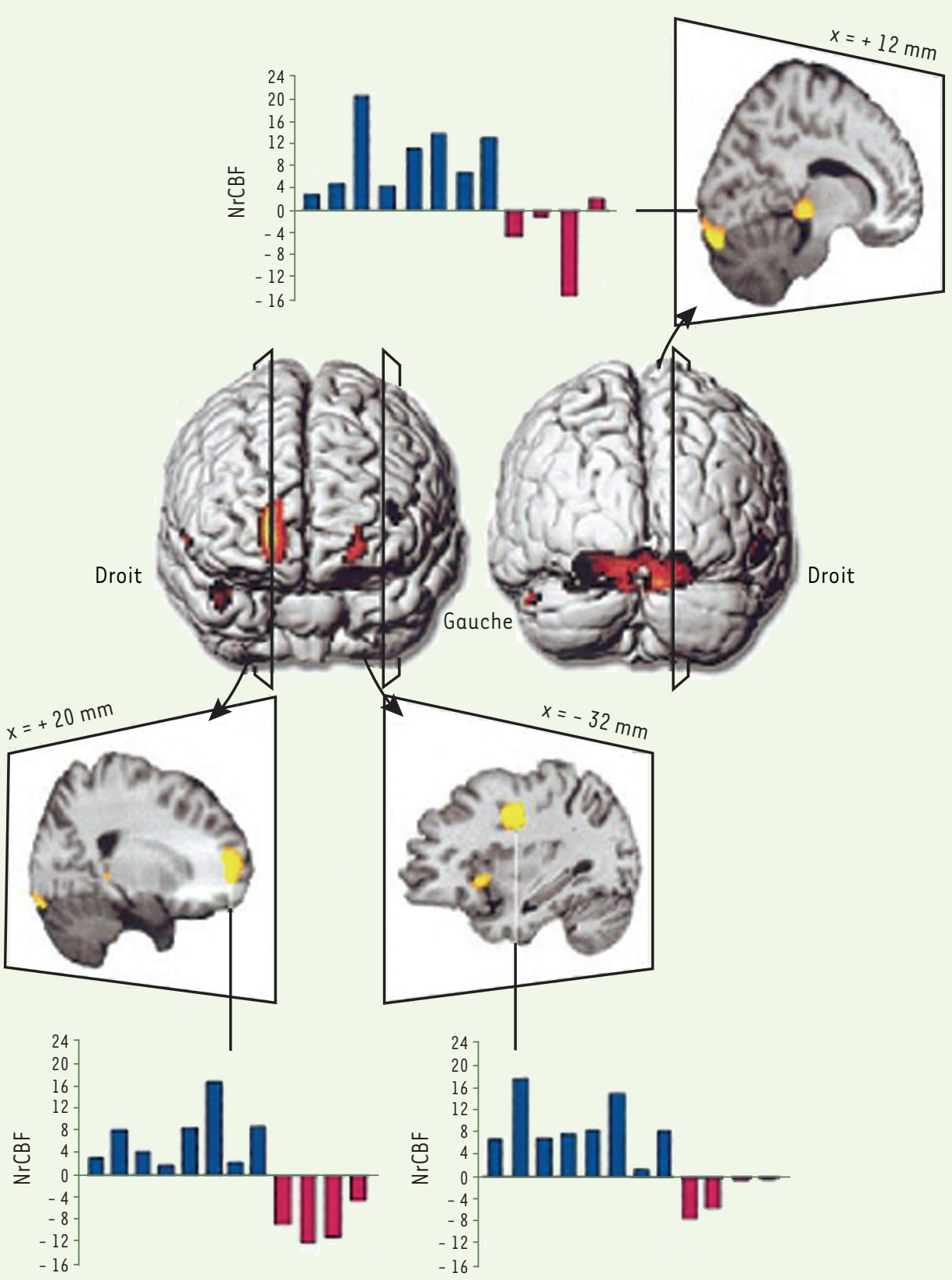

Figure 2. Neuro-imagerie de l'apprentissage de l'inhibition. L'une des activations cérébrales les plus importantes qui différencient l'apprentissage à l'inhibition du mode de raisonnement erroné par rapport à un apprentissage strictement logique est le cortex préfrontal ventromédian droit $(x=20)$, région paralimbique émotionnelle. L'analyse individuelle (c'est-à-dire la différence de débit sanguin cérébral régional normalisé individu par individu : NrCBF en anglais) confirme que cette région est activée chez les élèves qui accèdent à la réponse logique après l'apprentissage de l'inhibition (en bleu), donc après correction de l'erreur, alors qu'elle ne l'est pas chez ceux (en rouge) qui n'y accèdent pas (persistance dans l'erreur après un apprentissage logique «froid ») (d'après [21]).

variabilité neurocognitive intra-individuelle [17] provoquée ici par l'intervention pédagogique d'autrui (dont on trouve aussi des traces stabilisées à l'état de repos du cerveau [18]). Éducation et neurosciences peuvent donc se conjuguer ; il s'agit dans ce cas d'une forme de neuroscience humaine et sociale. L'imagerie cérébrale a ainsi apporté la visualisation biologique de l'effet pédagogique précédemment testé en psychologie expérimentale du raisonnement.

L'adulte, comme l'enfant, peut sans doute apprendre à inhiber les stratégies inadéquates de trois façons : soit par l'expérience propre à partir de ses échecs (démentis des prévisions, constat d'erreur), soit par imitation ou encore par des instructions pédagogiques venant d'autrui, comme dans l'étude réalisée. On pourrait dès lors développer à l'école une «pédagogie de l'inhibition » (au sens positif du terme) et du contrôle cognitif en général. Or l'école apprend plus systématiquement l'activation des connaissances, dans toutes les matières, que l'inhibition cognitive. Certains psychologues, comme Adèle Diamond au Canada, testent déjà avec succès des programmes expérimentaux de ce type dès l'école

d'abstraction neuronale par inhibition cognitive (de la réponse perceptive à la réponse logique) dans notre cerveau. C'est « le cerveau qui apprend en inhibant». En d'autres termes encore : c'est l'intelligence fluide qui se déclenche et s'observe chez l'élève à travers une maternelle : c'est le cas par exemple du programme «Tools of the mind » centré sur le contrôle cognitif [19]. Aux États-Unis, l'équipe de Posner a démontré, dans le même esprit, l'impact direct d'exercices de 
contrôle attentionnel sur le cerveau de jeunes enfants (4-6 ans) dans des régions sous forte influence génétique comme le cortex préfrontal et le cortex cingulaire antérieur qui lui est connexe [20]. Gènes et éducation sont ici conjugués.

\section{Le rôle de l'émotion et de la conscience réflexive}

Dans une étude suivante, nous avons cette fois directement comparé l'impact neuronal de l'apprentissage de l'inhibition (qui implique des mises en garde sur le danger du piège perceptif, sur l'erreur possible) et de l'apprentissage strictement rationnel et logique, que l'on peut considérer comme plus «froid » et scolaire. Les résultats ont indiqué : (1) au niveau comportemental, ce que l'on savait déjà, c'est-à-dire que seul l'apprentissage de l'inhibition est efficace dans les situations pièges étudiées (ce n'était pas la logique mentale en tant que telle qui manquait aux élèves), et (2) que l'une des activations cérébrales les plus importantes qui différencient ce type d'apprentissage de l'apprentissage logique est celle du cortex préfrontal ventromédian droit (CPVMD) [4, 12, 21]. II s'agit d'une région située dans «l'intimité » de notre cerveau droit, à l'avant (préfrontal), en bas (ventro) et au milieu (médian) - proche du système limbique qui est considéré comme «le cerveau des émotions ». Cette région paralimbique est connue, suite aux travaux du neuropsychologue Antonio Damasio, pour être impliquée dans les relations étroites entre émotion, sentiment de soi et raisonnement [22-24] : dans notre cas, l'émotion particulière liée au sentiment de s'être trompé, d'être tombé dans le piège mais de pouvoir néanmoins corriger son erreur de raisonnement, émotion complexe mêlant peur (de se tromper) et plaisir (de se corriger) que l'on a tous un jour ou l'autre ressentie. Elle est ici déclenchée et exercée, au niveau expérimental, par l'apprentissage du contrôle inhibiteur. L'analyse individuelle (c'est-à-dire la différence de débit sanguin cérébral régional individu par individu) confirme que le CPVMD est activé chez tous les élèves qui accèdent à la réponse logique après l'apprentissage de l'inhibition (donc après correction de l'erreur), alors qu'elle ne l'est pas chez ceux qui n'y accèdent pas (persistance dans l'erreur après un apprentissage logique «froid») (Figure 2). Sachant que la théorie de Damasio à propos du CPVMD est une théorie de la conscience réflexive, définie comme l'émotion liée au sentiment de soi au cours de l'activité cognitive, il est intéressant de noter qu'avant l'apprentissage de l'inhibition de l'erreur de raisonnement, les participants à notre expérience n'étaient pas conscients (n'avaient pas le sentiment) qu'ils commettaient une erreur de logique (ils pensaient tous répondre juste !), alors qu'après ils l'étaient (état dit de «conscience réflexive »). Ce phénomène de prise de conscience des erreurs de raisonnement par notre cerveau [25] est au cœur de toute la psychologie du développement, du bébé à l'adulte [14]. II l'est aussi, évidemment, en pédagogie [3].

\section{Neuropédagogie : un devoir d'humilité}

Après avoir exposé ces exemples de recherches en neuropédagogie expérimentale, il est important de rappeler, en conclusion, que les scientifiques, passionnés qu'ils sont, à juste titre, par leurs recherches et leurs découvertes, ont néanmoins en ce domaine un devoir d'humilité. D'abord parce qu'il s'agit de premières données encore très ponctuelles et partielles. Ensuite parce qu'il ne s'agit pas de dire aux professeurs des écoles ce qu'ils doivent faire dans leurs classes mais simplement de les informer (ainsi que les cadres de l'éducation et hommes politiques) des avancées technoscientifiques récentes (qui peuvent éventuellement confirmer des intuitions empiriques bien antérieures de certains professeurs) en matière d'apprentissages cognitifs et d'intervention pédagogique [2-6]. Ces découvertes, parfois contre-intuitives pour les professionnels (le rôle pédagogique positif de l'inhibition cognitive et son éducabilité cérébrale par exemple), peuvent avoir un impact dans le secteur sociétal de l'éducation, tout comme les neurosciences associées à la médecine ont déjà un fort impact dans le secteur de la santé. À l'image de la médecine, la pédagogie est un art qui doit aussi s'appuyer sur des connaissances scientifiques actualisées. En apportant des indications précises sur les capacités et les contraintes du «cerveau qui apprend », la psychologie et l'imagerie cérébrale peuvent aider à expliquer, au cas par cas, pourquoi certaines situations d'apprentissage sont efficaces, alors que d'autres ne le sont pas. Par exemple, les résultats exposés ici incitent, face aux blocages, erreurs ou difficultés robustes d'élèves particuliers, à tenir compte en pédagogie de deux mécanismes-clés, sans doute liés à des phénomènes éducatifs, sociaux et culturels plus complexes: l'inhibition de stratégies neurocognitives en compétition et l'émotion, associée au sentiment de soi, qui paraissent utiles au cerveau (parfois plus que la pure logique elle-même) pour corriger ses erreurs de raisonnement.

$\varepsilon n$ retour, le monde de l'éducation, très bien informé qu'il est de la pratique quotidienne - l'actualité de la pédagogie - peut suggérer des idées originales d'expérimentation en neuropédagogie. $\diamond$

\section{SUMMARY}

\section{The neural impact of cognitive training}

Tracing the connections from brain functions to education is a major goal of modern neuroscience. By providing insights into the abilities and constraints of the learning brain, neuroscience can help to explain why some learning environments work while others fail. The results presented here provide the first insights into neuropedagogy of reasoning. $\diamond$

\section{CONFLIT D'INTÉRÊTS}

L'auteur déclare n'avoir aucun conflit d'intérêts concernant les données publiées dans cet article. 


\section{RÉFÉRENCES}

1. Changeux JP. Du vrai, du beau, du bien : une nouvelle approche neuronale. Paris : Odile Jacob, $2008: 544 p$

2. Blakemore $S$, Frith $A$. The learning brain : lessons for education. Oxford : Blackwell, $2005: 216 \mathrm{p}$

3. Houdé 0.10 leçons de psychologie et pédagogie. Paris : PUF, $2006: 117 p$

4. Houdé 0 . First insights on neuropedagogy of reasoning. Thinking \& Reasoning 2007 ; 13: 81-9.

5. Posner M, Rothbart M. Educating the human brain. Washington : American Psychological Association, $2007: 263$ p.

6. Stern $\varepsilon$. Pedagogy meets neuroscience. Science $2005 ; 310: 745$.

7. Houdé 0 . Les 100 mots de la psychologie. Paris: PUF, $2008: 128 p$.

8. Kahn A. Un type bien ne fait pas ça... Morale, éthique et itinéraire personnel. Paris : Éditions du Nil, $2010: 278 \mathrm{p}$.

9. Raichle M, Fiez J, Videen T, et al. Practice-related changes in human brain functional anatomy during non-motor learning. Cerebral Cortex $1994 ; 4: 8-26$.

10. Posner M, Raichle M. L'esprit en images. Bruxelles : DeBoeck, $1998: 259 \mathrm{p}$.

11. Houdé 0 , Zago L, Mellet $\varepsilon$, et al. Shifting from the perceptual brain to the logical brain : the neural impact of cognitive inhibition training. J Cogn Neurosci $2000 ; 12: 721-28$.

12. Houdé 0 , Tzourio-Mazoyer N. Neural foundations of logical and mathematical cognition. Nat Rev Neurosci $2003 ; 4: 507-14$

13. Deary I, Penke L, Johnson W. The neuroscience of human intelligence differences. Nat Rev Neurosci $2010 ; 11: 201-11$

14. Houdé 0. La psychologie de l'enfant. Paris : PUF, 2004 : 128 p.

15. Posner M. Les neurosciences cognitives : synthèse de l'esprit et du cerveau. In : Dupoux $\varepsilon$, ed. Les langages du cerveau. Paris: Odile Jacob, 2002 : 401-14

16. Houdé 0 . Pedagogy, not (only) anatomy of reasoning. Trends Cogn Sci $2008 ; 12$ : 173-4

17. Houdé 0 . Beyond IQ comparisons : intra-individual training differences. Nat Rev Neurosci 2010 ; $11: 370$.
18. Mazoyer B, Houdé 0 , Joliot M, et al. Regional cerebral blood flow increases during wakeful rest following cognitive training. Brain Res Bull 2009; 80 : 133-8.

19. Diamond A, Barnett $W$, Thomas J, et al. Preschool program improves cognitive control. Science 2007 ; 318 : 1387-8.

20. Rueda M, Rothbart M, McCandliss B, et al. Training, maturation, and genetic influences on the development of executive attention. Proc Natl Acad Sci USA 2005 ; 102 : 14931-6.

21. Houdé 0 , Zago L, Crivello F, et al. Access to deductive logic depends on a right ventromedial prefrontal area devoted to emotion and feeling : Evidence from a training paradigm. Neuroimage 2001 ; 14 : 1486-92.

22. Damasio A. L'erreur de Descartes : la raison des émotions. Paris : Odile Jacob, 1995 : $365 \mathrm{p}$.

23. Damasio A. Le sentiment même de soi. Paris : Odile Jacob, 1999 : 380 p.

24. Damasio H, Grabowski T, Frank R, et al. The return of Phineas Gage : clues about the brain from the skull of a famous patient. Science $1994 ; 264$ : 1102-5.

25. Houdé 0 . Consciousness and unconsciousness of logical reasoning errors in the human brain. Behav Brain Sci $2003 ; 25: 341$.
TIRÉS À PART

0 . Houdé

\footnotetext{
CIIS

du 19 au 23 septembre 2011 PCR (Personne Compétente en Radioprotection)

à CLERMONT FERRAND (63) Module théorique

Secteurs "INDUSTRIE RECHERCHE" ou "MEDICAL"

du 20 au 22 septembre 2011 Initiation aux techniques de transplantation de cellules embryonnaires dans le cerveau de souris à POITIERS (86) nouveau-nées et adultes

du 21 au 23 septembre 2011 Analyse bioinformatique des séquences nucléiques et protéiques à PARIS (75)

Stage à la carte, nous consulter. RMN des macromolécules biologiques

à GIF-SUR-YVETTE (91)

du 3 au 7 octobre 2011 PCR (Personne Compétente en Radioprotection)

à CLERMONT FERRAND (63) Module pratique

Sources radioactives SCELLEES et NON SCELLEES

Secteur "MEDICAL"

du 7 au 10 novembre 2011 Phylogénie Moléculaire (Lyon)

à VILLEURBANNE (69)

du 7 au 10 novembre 2011 PCR (Personne Compétente en Radioprotection)

à CLERMONT FERRAND (63) RENOUVELLEMENT

Sources radioactives SCELLEES et NON SCELLEES

Secteur "MEDICAL"

Centre de ressources en formation

Un problème de formation particulier?

N'hésitez pas à nous consulter :

- par mail à ressources@cf.cnrs-gif.fr - par téléphone au 01.69.82.44.96

Catalogue, programmes et inscriptions : CNRS Formation Entreprises Avenue de la Terrasse Bât. 3191198 Gif-sur-Yvette Cedex Tél. : 0169824455 - Fax: 0169824489 http://cnrsformation.cnrs-gif.fr
} 


\section{5th p63/p73 Workshop at IARC in Lyon, France}

\section{Mon 12th - Wed 14th Sept, 2011}

Invited Speakers:

Aberdam D, Nice Attardi LD, stanford van Bokhoven $\mathrm{H}$, Nijmegen Bourdon JC, Dundee Caron de Fromentel C, Lyon Dobbelstein $\mathbf{M}$, Göttingen Doetsch V, Frankfurt Flores ER, Houston Green DR, Memphis Hainaut $P$, Lyon Kaplan D, Toronto Levine AJ, Princeton it Lu X, oxford Mantovani R, Milan Melino $G$, Leicester Mills AA, cold Spring Harbor Missero C, Naples Moll UM,-New York Nakagawara A, Chiba Pietenpol JA, Nashville Roop D, Denver Sabapathy $\mathbf{K}$, singapore Tomasini $\mathbf{R}$, Marseille Tommasino $M$, Lyon

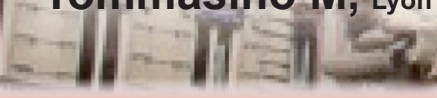

\section{Abstracts Submission Deadline: 15th June Early Registration Deadline: 1st July}

Contact the Secretariat: wrisez@iarc.fr or km179@le.ac.uk
Chairs:

Aberdam D

Caron de Fromentel C

Hainaut P

Melino G

Sabapathy K

Scientific Committee:

Flores ER

Levine AJ Lu $X$ Mantovani R McKeon $\mathbf{F}$ Mills AA Nakagawara A Kadakia MP

Pietenpol JA Prives $\mathrm{C}$

Secretariat: Michelle Wrisez Krupesh-Mistry Moll UM

\section{www.CDDconferences.eu} \& Disease

Cell Death 